\title{
VESSEL DIAMETER CHANGES DURING THE CARDIAC CYCLE
}

\author{
HEAN C. CHEN, VINOD PATEL, JUTTA WIEK, SALWAN M. RASSAM and EVA M. KOHNER \\ London
}

\begin{abstract}
SUMMARY
Retinal vessel diameter, which is an important parameter in blood flow measurement, is affected by pulsation during the cardiac cycle and by vasomotion. This project studied these changes by analysing three monochromatic fundus photographs taken in eight arbitrary parts of the cardiac cycle of 10 healthy subjects. It was found that the venous diameter decreased in early systole, increasing thereafter to a maximum level in early diastole and then declined towards end diastole. The maximum change of 4.82\% (between early systole and early diastole) $(p=0.03)$ represents a $9.83 \%$ change in volumetric blood flow. The arterial diameter peaked in mid-late systole, increasing by $3.46 \%(p=0.01)$; this represents a blood flow increase of $7.04 \%$. Vasomotion led to changes of $3.71 \%$ and $2.61 \%$ in arteries and veins respectively. It is concluded that for accurate measurement of retinal blood flow, fundus photographs should be taken synchronised with the electrocardiogram.
\end{abstract}

The retina is uniquely situated in allowing non-invasive direct visualisation of its circulatory system. This property means that the study of the retinal vasculature is of importance in furthering our understanding not only of retinal vessel pathology but also of normal vessel physiology. The study of retinal blood flow has been important in increasing our knowledge about the pathogenesis of various diseases, for example diabetic retinopathy, retinal occlusive diseases and glaucoma. ${ }^{1-3}$ Volumetric retinal blood flow is dependent on both the velocity of blood flow and the vessel diameter. With the advent of laser Doppler velocimetry there now exists an accurate non-invasive method for directly measuring retinal erythrocyte velocity in large retinal vessels. ${ }^{4}$

Volumetric flow is calculated as the product of the mean velocity of flow and the cross-sectional area of the vessel. As the latter is dependent upon the square function of the diameter, even small changes will lead to significant changes in the flow rate. Vessel diameter fluctuations are

Correspondence to: H. C. Chen, Diabetic Retinopathy Unit, Royal Postgraduate Medical School, Hammersmith Hospital, Du Cane Road, London W12 0NN, UK.

Eye (1994) 8, 97-103 C 1994 Royal College of Ophthalmologists likely to occur as a consequence of the pressure changes during each cardiac cycle (pulsatility), autoregulation and also as a result of vasomotion. ${ }^{5}$ Spontaneous venous pulsation of the central retinal vein is visible on direct ophthalmoscopy in a large proportion of the population; however, it is unknown whether smaller branches of the central retinal vein exhibit similar pulsations which would not be visible to the naked eye. Arterial blood flow being pulsatile in nature is likely to induce similar alterations in these vessels. Autoregulation is the ability of a blood vessel to alter its diameter either to maintain constant blood flow in the face of changing perfusion pressure or to alter flow to satisfy local metabolic requirements. ${ }^{6.7}$ Vasomotion is the spontaneous cyclical contraction and relaxation exhibited by some blood vessels; its presence has been demonstrated in the vasculature of the retina ${ }^{8}$ as well as of many other tissues, although the degree to which vasomotion occurs in the retina over a given period of time is unknown. ${ }^{9-13}$

Measurements of retinal blood flow that do not take these changes into account could be misleading. At rest, pulsatility and vasomotion are likely to account for most of the vessel diameter changes, with autoregulation playing an insignificant role. The aim of this study was to measure the variations in retinal vessel diameter in the healthy eye in the steady state. These vessel diameter changes may be altered in certain disease processes; the pattern of alteration could help further elucidate the pathogenesis or aid in diagnosis.

\section{SUBJECTS AND METHODOLOGY}

Ten healthy subjects were studied. The mean age was 34.6 years (range 29-57 years); there were 4 men and 6 women. All had normal blood pressure and intraocular pressure with the exception of one subject who had an intraocular pressure of $24 \mathrm{mmHg}$. Informed consent was obtained from all subjects in accordance with the Helsinki Declaration. The camera used was a standard Zeiss $30^{\circ}$ fundus camera (Zeiss, Oberkochen, Germany) using high-contrast Technical Pan film (Kodak, Rochester, NY, USA). The camera was fitted with a $570 \mathrm{~nm}$ filter to obtain monochromatic, red-free photographs which increases the resolution of the vasculature. ${ }^{14}$ 


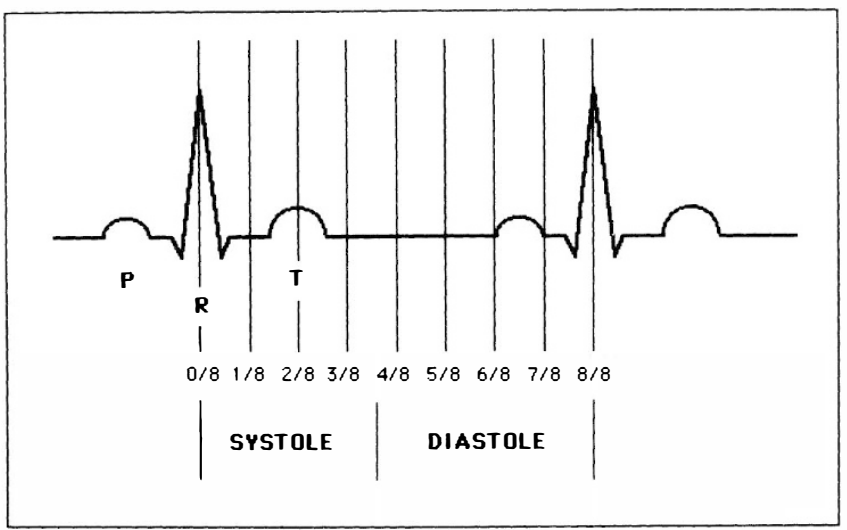

Fig. 1. Division of the events of the cardiac cycle into eighths.

The subjects were connected to an electrocardiogram (ECG) monitor (American Optical, Bedford, Mass., USA) with which the camera was synchronised through a timedelay switch. The time-delay switch was set to release the camera shutter at any chosen period after the $\mathrm{R}$ wave of the ECG to coincide with a particular part of the cardiac cycle;

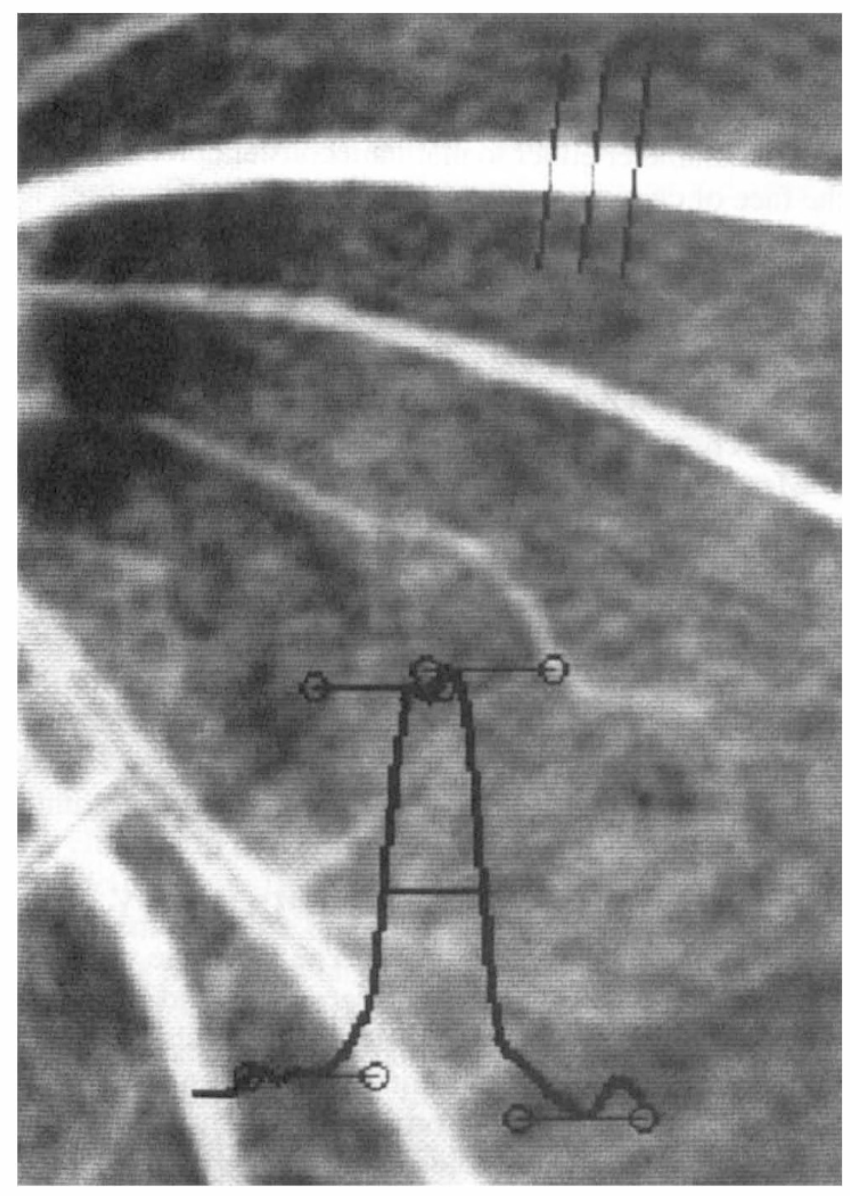

Fig. 2. The negative of a fundus photograph after digitisation by the Context Vision Image Analysis computer. It shows the computer-generated transmittance profile of the retinal vessel where it is marked by three perpendicular lines. The peak of this profile and the background values are indicated by the placement of cursors; with these levels marked the computer is then able to measure the width across the half-height of the profile, which is taken as the diameter. this delay period is a fraction of the duration of one cardiac cycle (R-R interval) which is dependent upon the subject's heart rate. The cardiac cycle was arbitrarily divided into eight parts and three photographs were taken in each of the parts. After the initial focusing requirements no further adjustments to the settings of the fundus camera were made and the subjects were required to remain still through the entire period.

The first set of photographs was taken at $1 / 8$ th of the cardiac cycle from the $\mathrm{R}$ wave, to coincide with early systole, through diastole, to 8/8th which coincides with the peak of the $\mathrm{R}$ wave. One cycle therefore extends between two $\mathrm{R}$ waves (0/8th to $8 / 8$ th) (Fig. 1). The photographs were analysed using the Context Vision Digital Image Analysis system (Linköping, Sweden). ${ }^{15}$ A high-resolution TV camera scans the negatives and relays the image to the image analysis computer which digitises and displays it on a $512 \times 512$ pixel array monitor. The measurement site on the vessel image is indicated by the placement of a cursor which cuts across the vessel perpendicularly. The computer then generates a transmittance profile of this site using the mean grey scale intensities from five loci distributed over 10 pixels on either side of the cursor. The background and peak intensity values of this profile are marked and from this the computer is able to determine its half-height; the width across the profile at its half-height is taken as the vessel diameter (Fig. 2). ${ }^{16}$ Three measurements were made of each photograph. As three photographs were taken of each part of the cardiac cycle, there were nine readings in total. The average of these nine readings was taken as the diameter for that part of the cardiac cycle. Care was taken to ensure that the measurements were made across the same point each time. Measurements were made of a major vein and artery within 1 disc diameter of the optic disc.

Comparisons were made of the vessel diameter at each part of the cardiac cycle with its value at the other seven parts. The overall variation through the cycle is expressed as the coefficient of variation of the cardiac cycle, which is the standard deviation expressed as a percentage of the mean diameter; this provides a measure of the degree of spread in the diameter value through the cycle.

In order to evaluate changes in the diameter resulting from vasomotion, the degree of variation between the measurements of the three photographs in each part of the cardiac cycle was examined. Similarly the spread of values between the three photographs was expressed as the coefficient of variation resulting from vasomotion.

Table I. Venous diameter

\begin{tabular}{lcc}
\hline & Venous diameters $(\mu \mathrm{m})$ & SEM \\
\hline $1 / 8$ & 143.04 & 7.98 \\
$2 / 8$ & 145.20 & 8.17 \\
$3 / 8$ & 147.30 & 7.90 \\
$4 / 8$ & 149.37 & 8.07 \\
$5 / 8$ & 149.93 & 8.84 \\
$6 / 8$ & 148.83 & 7.97 \\
$7 / 8$ & 147.12 & 7.54 \\
$8 / 8$ & 145.37 & 7.60 \\
\hline
\end{tabular}

Mean values are shown for each eighth of the cardiac cycle $(n=10)$. 


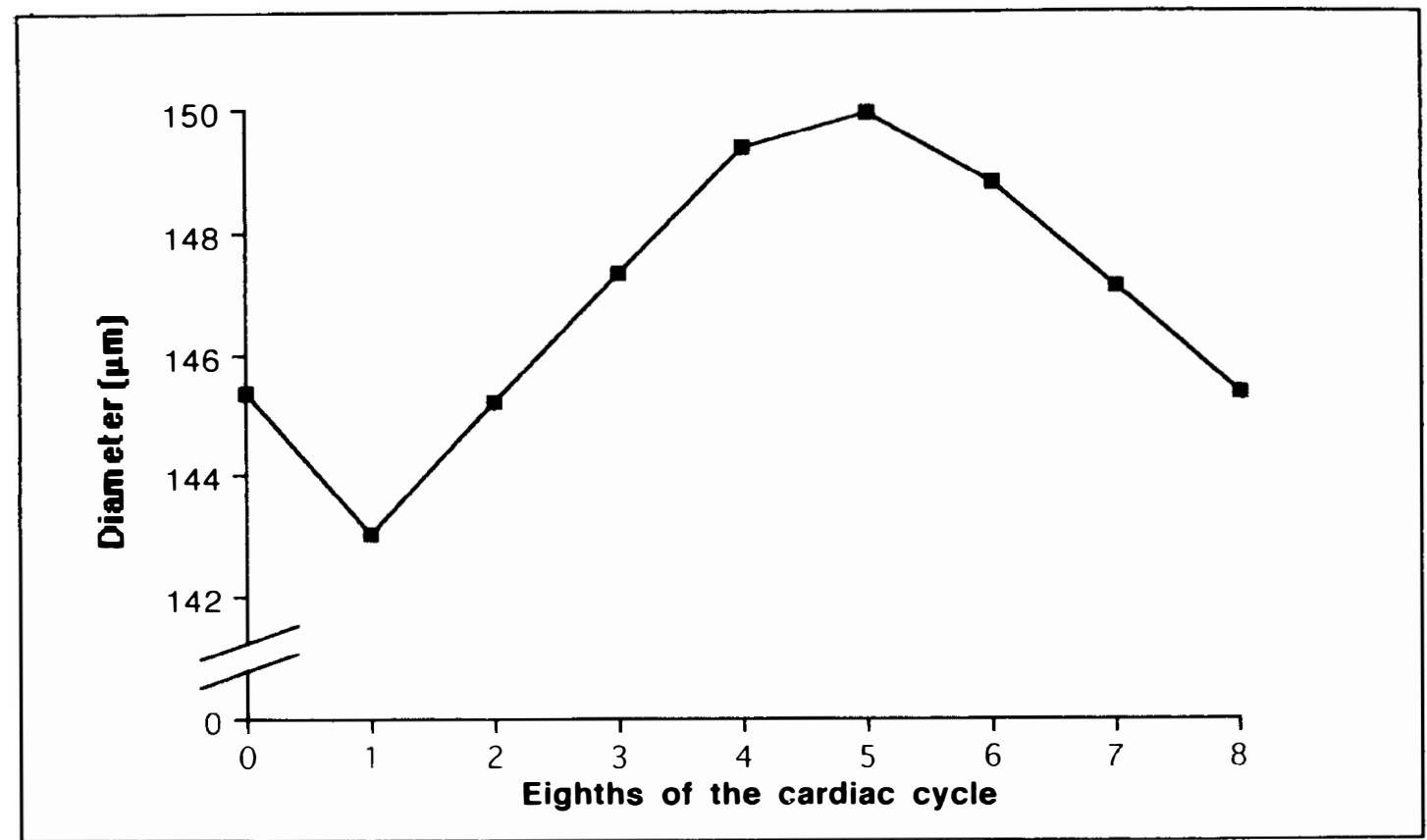

Fig. 3. Venous diameter means for each eighth of the cardiac cycle.

\section{Statistical Methods}

Analysis of variance was conducted on the data to ascertain whether significant intergroup differences existed. If this was found to be significant at $p<0.05$ further analysis was taken using a paired Student's $t$-test. Data are presented as the mean \pm standard error (unless stated otherwise) and a probability of 0.05 was considered to be statistically significant.

\section{RESULTS}

\section{Venous Diameter}

The venous diameter ranged from $105.9 \mu \mathrm{m}$ to $212.8 \mu \mathrm{m}$.
The mean values for each part of the cardiac cycle are presented in Table I. The analysis of variance showed that there were differences between the different parts of the cardiac cycle $(F=4.98, p=0.0002$, d.f. $=7)$. There was an initial reduction in the diameter at $1 / 8$ th (early systole) when compared with $0 / 8$ th (end diastole). This was followed by a gradual increase reaching a maximum level at 5/8th (early diastole) after which it started decreasing (Figs. 3, 4). The diameter measurement at 1/8th was smaller compared with the values at all other parts of the cardiac cycle, reaching statistical significance in the differences with $3 / 8$ th $(p=0.03)$, 4/8th $(p=0.02), 5 / 8$ th $(p=0.03)$ and 6/8th $(p=0.02)$. The same was true for the

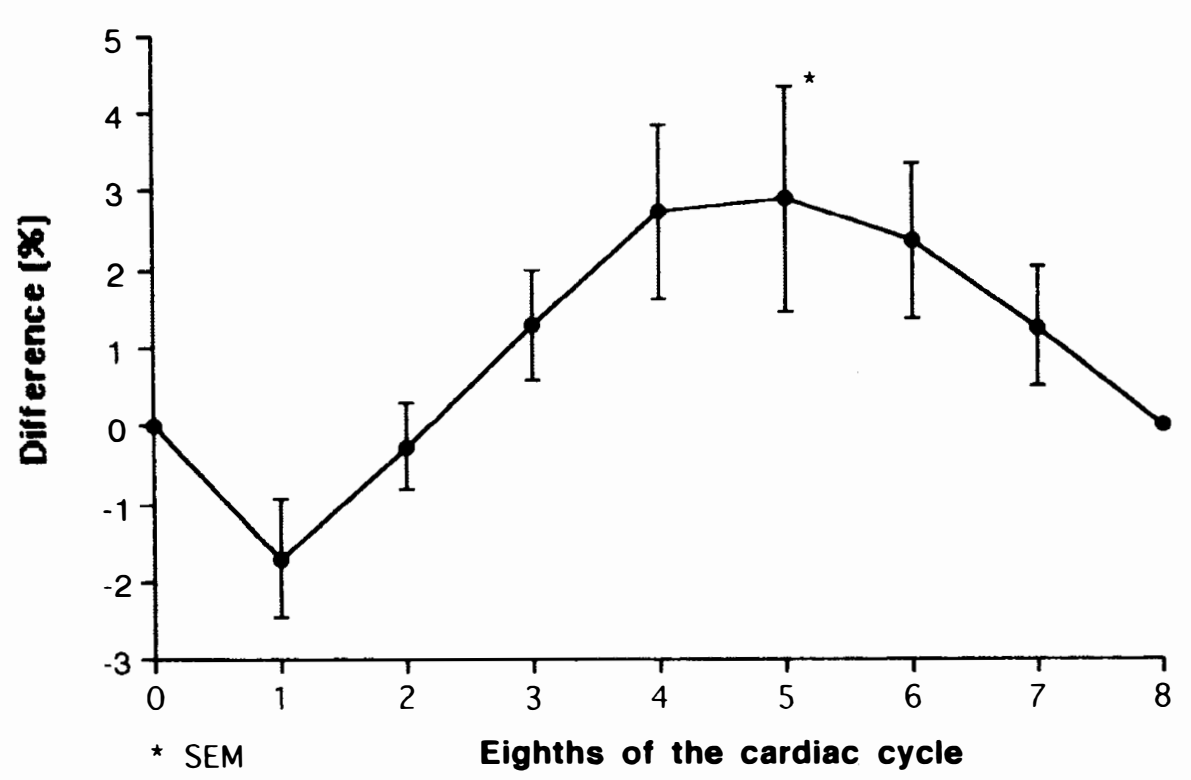

Fig. 4. Percentage difference of each eighth of the cardiac cycle from $0 / 8$ th (venous). 
Table II. Differences in venous diameter $(\%)$

\begin{tabular}{|c|c|c|c|c|c|c|c|c|}
\hline & $1 / 8$ & $2 / 8$ & $3 / 8$ & $4 / 8$ & $5 / 8$ & $6 / 8$ & $7 / 8$ & $8 / 8$ \\
\hline $1 / 8$ & - & & & & & & & \\
\hline $2 / 8$ & $+1.5 \%$ & - & & & & & & \\
\hline $4 / 8$ & $+4.4 \% *$ & $+2.9 \% *$ & $+1.4 \% *$ & - & & & & \\
\hline $5 / 8$ & $+4.8 \% *$ & $+3.3 \% *$ & $+1.8 \%$ & $+0.4 \%$ & - & & & \\
\hline $6 / 8$ & $+4.0 \% *$ & $+2.5 \% *$ & $+1.0 \%$ & $-0.4 \%$ & $-0.7 \% *$ & - & & \\
\hline $7 / 8$ & $+2.9 \%$ & $+1.3 \%$ & $-0.1 \%$ & $-1.5 \%$ & $-1.9 \%$ & $-1.1 \%$ & - & \\
\hline
\end{tabular}

The percentage differences between parts of the cardiac cycle as shown. (The cardiac cycle is divided into eight parts and the ' + ' and ' - ' signs indicate the change relative to the preceding part of the cycle.) $* p<0.05$.

measurement at $2 / 8$ th, which also reached statistical significance with $3 / 8$ th $(p=0.04), 4 / 8$ th $(p=0.01), 5 / 8$ th $(p=0.02)$ and $6 / 8$ th $(p=0.02)$. The measurements for 4/8th and 6/8th were significantly larger compared with $0 / 8$ th ( $p=0.04$ in both cases) (Table II). There is therefore an initial reduction of the vessel diameter in early systole (represented by $1 / 8$ th and $2 / 8$ th) relative to end diastole, followed by a gradual increase reaching a maximum level in early diastole, after which the diameter gradually decreases towards end diastole. The largest difference was between the values at $1 / 8$ th and $5 / 8$ th, which represented a change of $4.82 \%$; this corresponds to a $9.83 \%$ change in volumetric blood flow. The coefficients of variation for each individual subject through the cardiac cycle ranged from $0.91 \%$ to $4.50 \%$ with a mean of $2.18 \% \pm 0.51 \%$.

\section{Arterial Diameter}

The arterial diameter ranged between $82.9 \mu \mathrm{m}$ and $150.4 \mu \mathrm{m}$ (Table III). The analysis of variance showed that there were differences between the different parts of the cardiac cycle $(F=2.36, p=0.03$, d.f. $=7)$. The diameter increased from $1 / 8$ th (early systole), reaching a peak at $3 / 8$ th (mid-late systole), and then gradually declined towards $8 / 8$ th (end diastole) (Figs. 5, 6). The diameters at $2 / 8$ th and $3 / 8$ th were both significantly greater than the value at $1 / 8$ th $(p=0.04, p=0.01$ respectively); the $3 / 8$ th value was also significantly greater than that at $8 / 8$ th $(p=0.04)$ (Table IV). There is therefore an initial increase in the arterial diameter reaching a peak in mid to late systole and decreasing thereafter towards diastole. The largest difference was the increase from $1 / 8$ th to $3 / 8$ th, which represented a change of $3.46 \%$; this corresponds to a change in volumetric blood flow of $7.04 \%$. The coefficients of variation for each subject through the cardiac

Table III. Arterial diameter

\begin{tabular}{lcc}
\hline & Arterial diameter $(\mu \mathrm{m})$ & SEM \\
\hline $1 / 8$ & 115.61 & 6.36 \\
$2 / 8$ & 118.85 & 6.58 \\
$3 / 8$ & 119.34 & 6.40 \\
$4 / 8$ & 118.95 & 6.61 \\
$5 / 8$ & 117.91 & 6.78 \\
$6 / 8$ & 118.91 & 6.43 \\
$7 / 8$ & 117.43 & 6.48 \\
$8 / 8$ & 116.11 & 6.41 \\
\hline
\end{tabular}

Mean values are shown for each eighth of the cardiac cycle $(n=10)$. cycle ranged from $1.24 \%$ to $4.64 \%$ with a mean of $2.32 \% \pm 0.38 \%$.

\section{Vasomotion}

Fluctuations in retinal vessel diameter may be due to pulsatility (secondary to changes during the cardiac cycle), vasomotion or autoregulation. By examining changes between the three photographs taken in one part of the cardiac cycle, the element of pulsatility is eliminated; similarly autoregulatory changes are unlikely to contribute significantly to any changes in the period of time over which the photographs were taken, given that the patients were in a relatively steady state. The difference between the three photographs obtained from each part of the cardiac cycle (inter-photographic variation) was therefore taken as a measure of vasomotion. Although inter-photographic variation may result from methodological discrepancies, this possible source of variation was kept to a minimum by maintaining a constant camera-to-eye distance with no further adjustments to the camera settings once photography had commenced; it was also stressed to the subjects the importance of steady fixation on a fixed target with their contralateral eye and the photographs obtained were generally of a high standard.

The mean inter-photographic variations were $3.71 \% \pm 0.30 \%$ and $2.61 \% \pm 0.24 \%$ for arteries and veins respectively; the corresponding coefficients of variation were $1.90 \% \pm 0.15 \%$ for arteries and $1.36 \% \pm 0.12 \%$ for veins. Since the coefficient of variation for the measurement technique (intra-photographic variation, which is the variation between the three measurements on any individual photograph) was only $0.16 \% \pm 0.02 \%$, the inter-photographic values are significant.

\section{DISCUSSION}

This study has shown that both retinal arterial and venous diameters undergo change concomitant with the events of the cardiac cycle.

The arterial change is perhaps to be expected as a consequence of the pulse wave entering the eye. The arterial diameter reaches a maximum in mid systole, which is just after the period of peak aortic pressure; subsequently, as arterial pressure decays from its peak, the vessel diameter diminishes towards end diastole. ${ }^{17}$ 
Table IV. Differences in arterial diameter

\begin{tabular}{|c|c|c|c|c|c|c|c|c|}
\hline & $1 / 8$ & $2 / 8$ & $3 / 8$ & $4 / 8$ & $5 / 8$ & $6 / 8$ & $7 / 8$ & $8 / 8$ \\
\hline $1 / 8$ & - & & & & & & & \\
\hline $2 / 8$ & $+2.6 \% *$ & - & & & & & & \\
\hline $3 / 8$ & $+3.2 \% *$ & $+0.4 \%$ & - & & & & & \\
\hline $4 / 8$ & $+2.9 \%$ & $+0.1 \%$ & $-0.3 \%$ & - & & & & \\
\hline $5 / 8$ & $+2.0 \%$ & $-0.8 \%$ & $-1.2 \%$ & $-0.9 \%$ & - & & & \\
\hline $6 / 8$ & $+2.1 \%$ & $-0.6 \%$ & $-1.0 \%$ & $-0.7 \%$ & $+0.2 \%$ & - & & \\
\hline $7 / 8$ & $+1.5 \%$ & $-1.2 \%$ & $-1.6 \%$ & $-1.3 \%$ & $-0.4 \%$ & $-0.6 \%$ & - & \\
\hline $8 / 8$ & $+0.4 \%$ & $-2.3 \% *$ & $-2.7 \%$ & $-2.4 \%$ & $-1.5 \%$ & $-1.7 \%$ & $-1.1 \%$ & - \\
\hline
\end{tabular}

The percentage differences between parts of the cardiac cycle are shown. (The cardiac cycle is divided into eight parts and the ' + ' and '-' signs indicate the change relative to the preceding part of the cycle.) $* p<0.05$.

In early systole, as a result of a greater volume of blood entering its vascular tree, the volume of the globe increases by up to $4 \%$, which in turn increases the intraocular pressure by $1-2 \mathrm{mmHg} .{ }^{18}$ The diameter of a retinal vein, which is a thin-walled structure, is maintained by a balance of two main forces: the outwardly directed intravascular pressure and the opposing intraocular pressure. These two pressures are normally very similar and small fluctuations in either may lead to large changes in the transmural or distending pressure (intravascular pressure minus the intraocular pressure); it is this distending pressure which is responsible for keeping the vessel open. The increase in intraocular pressure during systole will lead to a reduction in the distending pressure. This therefore results in a passive reduction of the venous diameter and is in accordance with the law of Laplace which states that the distending pressure in a hollow object is proportional to its wall tension and radius.

The subsequent increase in venous diameter is due to a combination of the systolic increase in blood flow reaching the venous part of the vascular tree and the intraocular pressure returning to its diastolic level. The intra-arterial pressure, which is considerably higher than both the intra- venous and intraocular pressures, will not be subject to small alterations in the intraocular pressure; the artery is also a vessel with a thicker wall.

The results of this study are largely in agreement with the work by Delori et al. ${ }^{19}$ as to the times at which vessel diameter is greatest and smallest, although they found the change through the cycle to be smaller. They expressed their results in terms of vessel diameter pulsatility (VDP) which was defined as the difference between the maximal and minimal vessel diameter expressed as a percentage of the pulse-averaged diameter; absolute values were not presented. They detected mean VDP values of $2.3 \pm 0.7 \%$ (SD) and $2.9 \pm 1.9 \%(\mathrm{SD})$ for arteries and veins respectively. These values are smaller than those of the present study, which are $7.22 \pm 3.9 \%$ (SD) and $6.45 \pm 4.67 \%(\mathrm{SD})$ for arteries and veins respectively. They did, however, employ a different mode of measurement using scanning fundus reflectometry.

The magnitude of vasomotion in human retinal vessels has, to our knowledge, not previously been documented. Riva et al. ${ }^{20}$ have previously shown a periodic variation in the blood flow of the optic nerve head, retina and choroid at frequencies in the range of 2-4 per minute in miniature

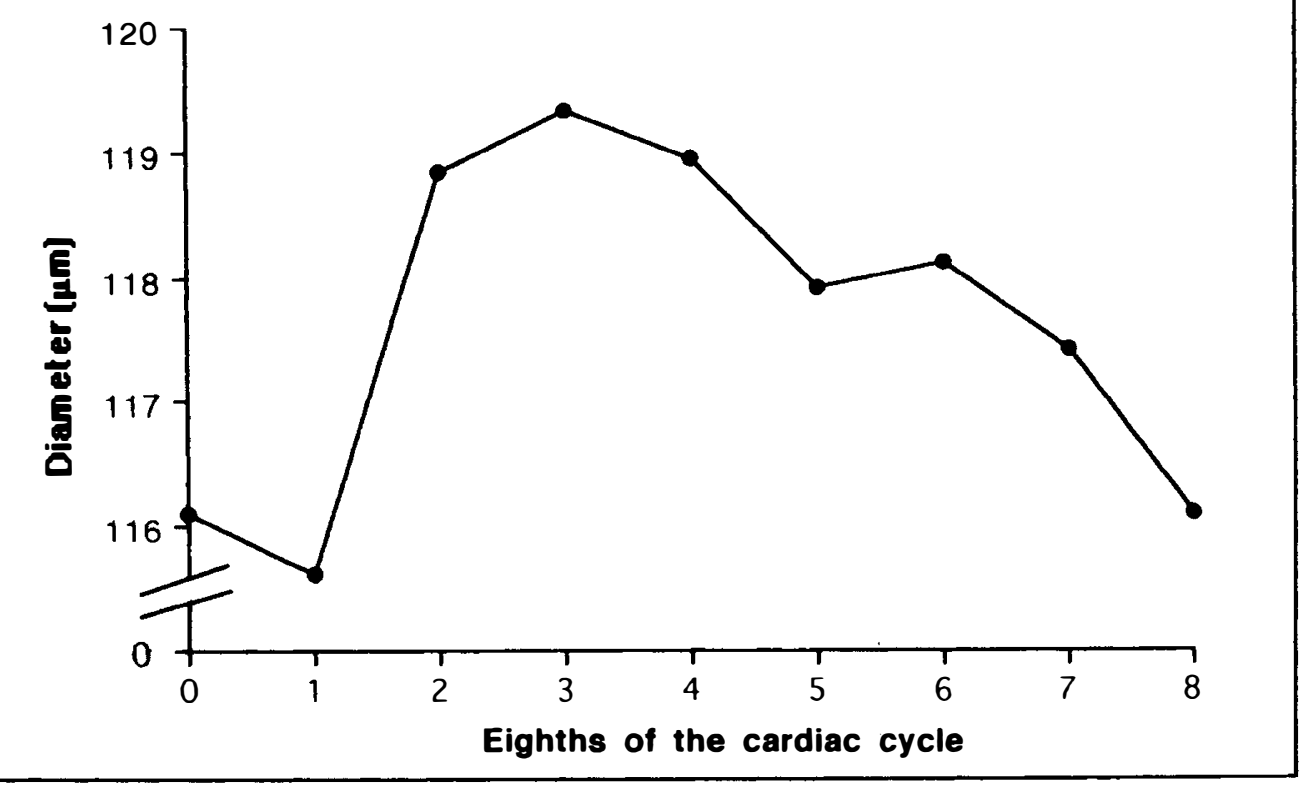

Fig. 5. Arterial diameter means for each eighth of the cardiac cycle. 


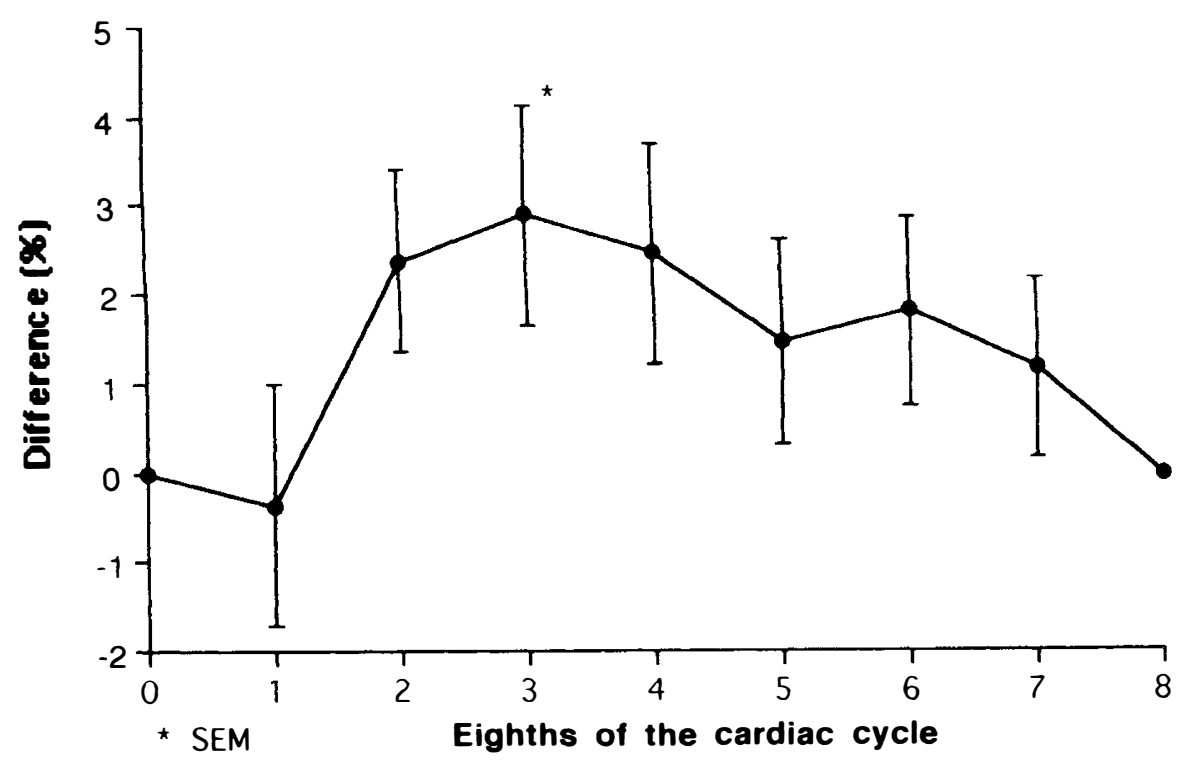

Fig. 6. Percentage difference of each eighth of the cardiac cycle from 0/8th (arterial).

pigs which they attributed to vasomotion. This phenomenon of spontaneous cyclical relaxation and contraction is well recognised in many tissues and may be altered by humoral, metabolic, physical and nervous stimuli. ${ }^{5}$ As retinal vessels do not possess autonomic innervation, the local factors are therefore likely to play an important role in modulating the frequency and size of vasomotion; these same factors are also likely to be important in retinal vessel autoregulation, which as earlier stated is a separate phenomenon. The degree of vasomotion may therefore be compromised in conditions where the local environment is altered. This alteration in vasomotive ability may be of fundamental importance in the underlying pathogenic mechanisms - for example a vessel the diameter of which oscillates possesses a notably lower resistance than one with a diameter that is static. ${ }^{11}$ The vascular endothelium plays an important role in the modulation of vasomotion and is affected by conditions such as diabetes and hypertension; vasomotion is also thought to play a significant role in the pathogenesis of conditions such as unstable angina and variant angina. ${ }^{21,22}$

Fig. 7 summarises the variations in diameter accounted for by the cardiac cycle, vasomotion and errors arising from the measurement technique. This emphasises the importance of coupling retinal photography with the ECG for accurate comparisons of retinal vessel diameters. We have shown that an error of up to $7.04 \%$ and $9.83 \%$ variation in volumetric arterial and venous blood flow measurements respectively may occur by not taking this into account. It is therefore important, especially when serial

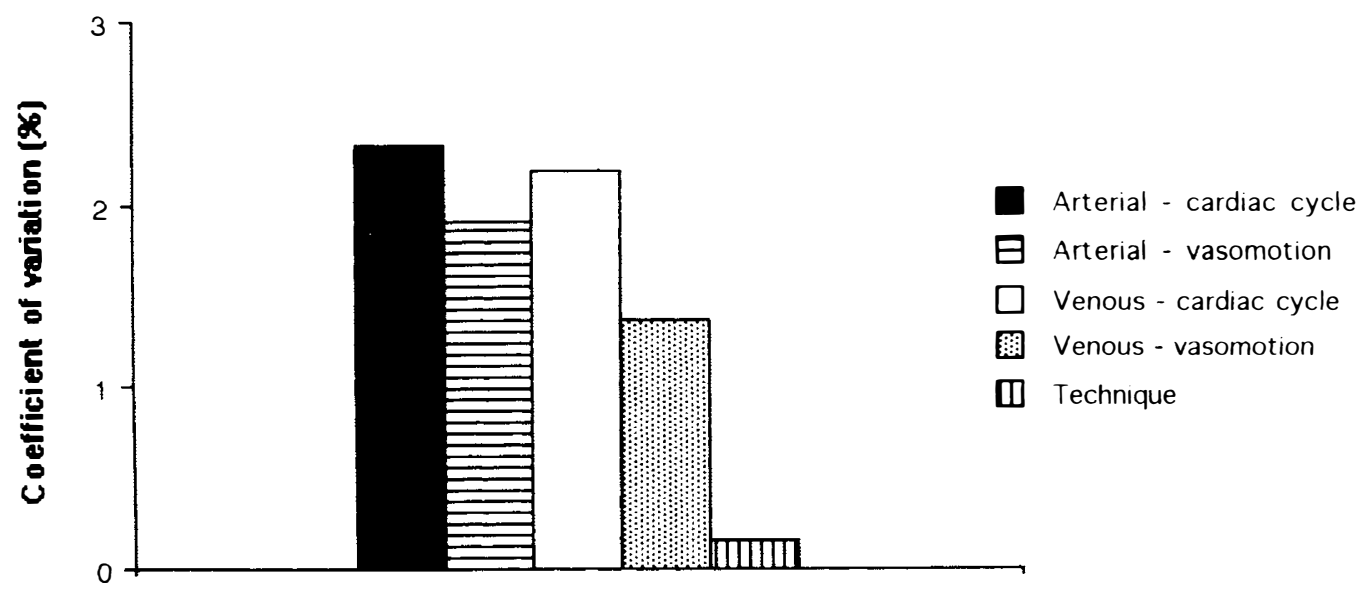

Fig. 7. Diameter variation for artery and vein resulting from cardiac cycle changes, vasomotion and measurement technique. 
photographis are taken, to study changes over time, that these photographs are taken synchronised with the ECG.

Apart from the above, retinal vessel diameter changes in the cardiac cycle may be altered in disease states and the pattern of variation may suggest possible pathogenic mechanisms. In conditions in which the intravenous pressure is raised, for example impending central retinal vein occlusion and raised intracranial pressure, there will be a diminution of the venous pulsatility, whereas a reduction in the perfusion pressure as would occur, for example, with a deficient carotid supply would lead to reduced arterial pulsatility. Examining retinal vessel diameter changes in the cardiac cycle may therefore provide another avenue for studying diseases which potentially compromise the circulation.

Key words: Blood flow, Cardiac cycle, Fundus photographs. Pulsation, Retinal vessel diameter, Vasomotion.

\section{REFERENCES}

1. Patel V, Rassam S, Newsom R, Wiek J, Kohner E. Retinal blood flow in diabetic retinopathy. BMJ 1992;305:678-83.

2. Grunwald JE, Riva CE, Stone RA, Keates EU, Petrig BL. Retinal autoregulation in open-angle glaucoma. Ophthalmology 1984;91:1690-4.

3. Fujino T, Curtin VT, Norton EWD. Experimental central retinal vein occlusion: a comparison of intraocular and extraocular occlusion. Arch Ophthalmol 1969;81:395-406.

4. Riva CE, Grunwald JE, Sinclair SH, O'Keefe K. Fundus camera based retinal LDV. Appl Optics 1981;20:117-20.

5. Wise G, Dollery C, Henkind P. Physiologic principles. In: The retinal circulation. New York: Harper \& Row, 1971: 83-117.

6. Johnson PC. Review of previous studies and current theories of autoregulation. Circulation Res 1964;14/15(Suppl.1): 2-9.

7. Guyton AC. Local control of blood flow by the tissues, and nervous and humoral regulation. In: Textbook of medical physiology. Philadelphia: Saunders, 1981;232-45.

8. Braun RD, Linsenmeier RA, Yancey CM. Spontaneous fluc- tuations in oxygen tension in the cat retina. Microvasc Res 1992;44:73-84.

9. Nicholl PA, Webb RL. Vascular pattern and active vasomotion as determiners of flow through minute vessels. Angiology 1955;6:291-310.

10. D'Agrosa LS. Patterns of venous vasomotion in the bat wing. Am J Physiol 1970;218:530-5.

11. Colantuoni A, Bertuglia S, Intaglietta M. Quantitation of rhythmic diameter changes in arterial microcirculation. Am J Physiol 1984;246:H508-17.

12. McAlpin RN. Contribution of dynamic vascular wall thickening to luminal narrowing during coronary arterial constriction. Circulation 1980;61:296-301.

13. Intaglietta M, Endrich BA. Experimental and quantitative analysis of microcirculatory water exchange. Acta Physiol Scand Suppl 1979;463:59-66.

14. Delori FC, Gragoudas ES, Francisco R, Pruett RC. Monochromatic ophthalmoscopy and fundus photography. Arch Ophthalmol 1977:95:861-8.

15. Newsom RBS, Paul MS, Rassam SMB, Jagoe R, Kohner EM. Retinal vessel measurement: comparison between observer and computer driven methods. Graefes Arch Clin Exp Ophthalmol 1992;230:221-5.

16. Brinchmann HO, Engvold O. Microphotometry of the blood column and the light streak on retinal vessels in fundus photographs. Acta Ophthalmol (Cophenh) 1986;95:861-8.

17. Ganong WF. The heart as a pump. In: Review of medical physiology. Los Altos: Lange Medical Publications, 1985: 459-69.

18. Alm A. Ocular circulation. In: Hart WM Jr, editor. Adler's physiology of the eye. St Louis: Mosby Year Book, 1992: 198-227.

19. Delori FC, Fitch KA. Variation of retinal vessel diameter with cardiac cycle [abstract]. Invest Ophthalmol Vis Sci 1988:29: 178.

20. Riva CE, Pournaras CJ, Poitry-Yamate CL, Petrig BL. Rhythmic changes in velocity, volume and flow of blood in the optic nerve head tissue. Microvasc Res 1990;40:36-45.

21. Harrison DG, Kurz MA, Quillen JE, Sellke FW, Mugge A. Normal and pathophysiologic considerations of endothelial regulation of vascular tone and their relevance to nitrate therapy. Am J Cardiol 1992;70:B 11-7.

22. Pepine CJ. Daily life ischemia and nitrate therapy. Am J Cardiol 1992;70:B54-63. 